\title{
Genes associated with agronomic traits in non-heading Chinese cabbage identified by expression profiling
}

\author{
Xiaoming Song ${ }^{\dagger}$, Ying Li ${ }^{\dagger}$, Tongkun Liu, Weike Duan, Zhinan Huang, Li Wang, Huawei Tan and Xilin Hou
}

\begin{abstract}
Background: The genomes of non-heading Chinese cabbage (Brassica rapa ssp. chinensis), heading Chinese cabbage (Brassica rapa ssp. pekinensis) and their close relative Arabidopsis thaliana have provided important resources for studying the evolution and genetic improvement of cruciferous plants. Natural growing conditions present these plants with a variety of physiological challenges for which they have a repertoire of genes that ensure adaptability and normal growth. We investigated the differential expressions of genes that control adaptability and development in plants growing in the natural environment to study underlying mechanisms of their expression.

Results: Using digital gene expression tag profiling, we constructed an expression profile to identify genes related to important agronomic traits under natural growing conditions. Among three non-heading Chinese cabbage cultivars, we found thousands of genes that exhibited significant differences in expression levels at five developmental stages. Through comparative analysis and previous reports, we identified several candidate genes associated with late flowering, cold tolerance, self-incompatibility, and leaf color. Two genes related to cold tolerance were verified using quantitative real-time PCR.
\end{abstract}

Conclusions: We identified a large number of genes associated with important agronomic traits of non-heading Chinese cabbage. This analysis will provide a wealth of resources for molecular-assisted breeding of cabbage. The raw data and detailed results of this analysis are available at the website http://nhccdata.njau.edu.cn.

Keywords: Non-heading Chinese cabbage, Expression profile, Differentially expressed genes, Protein function annotation, Chromosome distribution, Agronomic traits

\section{Background}

Brassica rapa L. plants have a rich morphological and genetic diversity, comprising many plant subspecies that humans farm on an enormous scale worldwide. Examples of agriculture crops include turnip, field mustard, and Chinese cabbage. Non-heading Chinese cabbage (Brassica rapa ssp. chinensis), with its five varieties, is an excellent model to study the genetics and mechanisms underlying phenotypic diversity. Of particular interest are its flowering and self-incompatibility characteristics. In general, a higher plant's conversion from vegetative growth to flowering is a

\footnotetext{
* Correspondence: hxl@njau.edu.cn

${ }^{\dagger}$ Equal contributors

State Key Laboratory of Crop Genetics and Germplasm Enhancement/Key Laboratory of Biology and Germplasm Enhancement of Horticultural Crops in East China, Ministry of Agriculture, Nanjing Agricultural University, Nanjing East China, Minist
}

C Biomed Central

(C) 2014 Song et al.; licensee BioMed Central Ltd. This is an Open Access article distributed under the terms of the Creative Commons Attribution License (http://creativecommons.org/licenses/by/2.0), which permits unrestricted use, distribution, and reproduction in any medium, provided the original work is properly credited. The Creative Commons Public Domain Dedication waiver (http://creativecommons.org/publicdomain/zero/1.0/) applies to the data made available in this article unless otherwise stated. pivotal point in ontogeny and decides the timing and quality of its reproduction. Floral induction has become a focus of research in Brassica vegetables [1-3], and non-heading Chinese cabbage is an important tool in this regard.

Recent progress in molecular biology techniques has revealed that floral induction is regulated through long-day, autonomous, vernalization, and gibberellin-dependent genetic pathways [4-6]. Early- and late-flowering mutants have been identified in the model plant Arabidopsis, and many key genes controlling flowering have also been isolated in other plants, genes that include $F L C, L F Y, F T$, and SOC1 [6-8]. However, there were few reports about the flowering of the non-heading Chinese cabbage, or the genes that regulate flowering.

Self-incompatibility is a genetic mechanism that prevents self-pollination (selfing) and inbreeding with close relatives. It promotes the divergence of species; any allele 
that is rare in the population has an advantage if residing in a plant that cannot self-fertilize. In angiosperms selffertilization is prevented by the chemical recognition of pollen by pistils, which depends on self-sterile $(S)$-alleles in pollen or stigma, and has evolved independently at least three times [9]. However, the shift from outcrossing to selfing is a common evolutionary trend in higher plants related to the loss of function under natural selection of the $S$-alleles in pollen or stigma [10]. Thus, the plant self-incompatibility system is an excellent model for understanding the variability in S loci. Shifts between outcrossing and selfing and frequency-dependent selection leads to the long-term maintenance of many alleles with different incompatibility types [11], and alleles thus become widely dispersed throughout different populations of species $[9,12]$.

In non-heading Chinese cabbage production, the selfincompatibility system is relied on for breeding, and also makes the plant a model system for studying reproductive biology and balancing selection [13]. With the recent development of next-generation, high-throughput sequencing technologies, the expression profiles of many species have been extensively studied. In addition, digital gene expression tag profiling has been used to study changes in gene expressions [14,15], giving a comprehensive snapshot of changes in mRNA expression that occur during biological processes. Expression levels can be calculated by the number of detected tags, and this information can facilitate our understanding of plant genetics and developmental mechanisms.

As of yet there has been no report of an expression profile for non-heading Chinese cabbage. To identify differentially expressed genes (DEGs) among different non-heading Chinese cabbage accessions in their natural environment, we conducted an expression profile analysis for plants growing under non-controlled conditions. Among the five varieties of non-heading Chinese cabbage produced in China, approximately $80 \%$ is Pak-choi (also known as bokchoy). Therefore, we chose three accessions of Pak-choi (NHCC001, NHCC002, and NHCC004) for investigating the differences in expression profiles, at five important developmental stages. Relative to the accessions NHCC001 and $\mathrm{NHCCO02,} \mathrm{NHCCOO4} \mathrm{bolts} \mathrm{and} \mathrm{flowers} \mathrm{later,} \mathrm{and}$ NHCC002 is the only one that is self-incompatible. The leaf color of NHCCOO2 is lighter than that of NHCCO01 and NHCC004. We systematically and comprehensively evaluated the expression profiles of these accessions, to identify the DEGs at the five development stages, to analyze their expression patterns, and to identify candidate genes associated with important agronomic traits.

\section{Results and discussion}

\section{Expression profiling of non-heading Chinese cabbage}

We used high-throughput sequencing to survey the gene expression patterns of three non-heading Chinese cabbage cultivars (NHCC001, NHCC002, and NHCC004) at five development stages (five leaf, rosette, adult, bolting, and flowering). A total of 55.45 million reads of raw tags were sequenced. After filtering, we obtained approximately 17.47, 17.97, and 17.77 million reads of clean tags for NHCC001, NHCC002, and NHCC004, respectively, of all five developmental stages combined (Additional file 1: Table S1). In these clean tags, $73.61 \%$ (12.85 million), 69.73\% (12.53 million), and 68.18\% (12.12 million) reads from NHCC001, NHCC002, and NHCC004, respectively, could be mapped to non-heading Chinese cabbage genes modeled from the NHCCO01 draft genome, and 63.00\% (11.00 million), $60.37 \%$ (10.84 million), and $57.98 \%$ (10.30 million) reads from the respective accessions could be mapped to unique genes (Table 1).

A total of 29101 genes were detected at the five developmental stages of the three non-heading Chinese cabbage accessions (Additional file 2: Figure S1). There were 15 251, 13 316, and 5869 expressed genes shared by all five development stages in NHCC001, NHCC002, and NHCC004, respectively. We also conducted a study of gene expression in the different accessions at each stage. A total of 13546 , 15 281, 15 029, 16 333, and 12704 co-expressed genes in all three accessions were detected in the leaf, rosette, adult, bolting, and flowering stages, respectively (Additional file 2: Figure S2).

\section{Identification of DEGs in non-heading Chinese cabbage}

A total of 15830 unique genes were found to be differentially expressed among the three accessions in the five development stages (Additional file 1: Table S2). The number of DEGs per accession or developmental stage is shown as a Venn diagram and in tables (Figure 1, Additional file 2: Figure S3, Additional file 1: Table S3, Table S4). To gain insights into the DEGs, we conducted a chi-squared test, and the $P$ values were corrected using the false discovery rate (Additional file 1: Table S5). The upregulated and downregulated genes are shown in a scatterplot (Additional file 2: Figure S4).

We used the Cluster program (http://bonsai.hgc.jp/ mdehoon/software/cluster/software.htm) to identify subgroups in the gene expression profiles that shared common features and had similar expression levels. We hypothesized that DEGs gathered in one group might have similar functions, or be involved in the same metabolic processes. In our analysis, clusters were plotted according to the DEG expression values. In Cluster, the DEGs that had similar expression levels were clustered together. By using these clusters, we could infer the function of newly identified genes according to the known genes in the same cluster, such as the cluster of flowering and self-incompatibility candidate genes (Figure 2, Additional file 2: Figure S5). 
Table 1 Alignment of the expression profile read to the genome of non-heading Chinese cabbage

\begin{tabular}{|c|c|c|c|c|c|c|c|c|}
\hline $\begin{array}{l}\text { Sample } \\
\text { accession }\end{array}$ & $\begin{array}{l}\text { Developmental } \\
\text { stage }\end{array}$ & $\begin{array}{l}\text { Total } \\
\text { reads }\end{array}$ & $\begin{array}{l}\text { Mapped } \\
\text { reads }\end{array}$ & $\begin{array}{l}\text { Uninq mapped } \\
\text { reads }\end{array}$ & $\begin{array}{l}\text { Mapped } \\
\text { ratio (\%) }\end{array}$ & $\begin{array}{l}\text { Uniq mapped } \\
\text { ratio }(\%)\end{array}$ & $\begin{array}{l}\text { Average mapped } \\
\text { ratio }(\%)\end{array}$ & $\begin{array}{l}\text { Average uniq } \\
\text { mapped ratio (\%) }\end{array}$ \\
\hline \multirow[t]{5}{*}{$\mathrm{NHCCO01}$} & Seedling & 3416587 & 2698481 & 2348539 & 78.98 & 68.74 & 73.61 & 63.00 \\
\hline & Rosette & 3524418 & 2530708 & 2226602 & 71.80 & 63.18 & & \\
\hline & Adult & 3606773 & 2607373 & 2118040 & 72.29 & 58.72 & & \\
\hline & Bolting & 3426539 & 2642635 & 2254999 & 77.12 & 65.81 & & \\
\hline & Flowering & 3500555 & 2375503 & 2049847 & 67.86 & 58.56 & & \\
\hline \multirow[t]{5}{*}{$\mathrm{NHCCOO2}$} & Seedling & 3421189 & 2542362 & 2258193 & 74.31 & 66.01 & 69.73 & 60.37 \\
\hline & Rosette & 3622963 & 2571338 & 2280414 & 70.97 & 62.94 & & \\
\hline & Adult & 3648700 & 2604740 & 2164281 & 71.39 & 59.32 & & \\
\hline & Bolting & 3692773 & 2713241 & 2320313 & 73.47 & 62.83 & & \\
\hline & Flowering & 3587470 & 2099277 & 1820960 & 58.52 & 50.76 & & \\
\hline \multirow[t]{5}{*}{$\mathrm{NHCCO} 4$} & Seedling & 3488808 & 1985099 & 1765494 & 56.90 & 50.60 & 68.18 & 57.98 \\
\hline & Rosette & 3396774 & 2434589 & 2115424 & 71.67 & 62.28 & & \\
\hline & Adult & 3706078 & 2642722 & 2136807 & 71.31 & 57.66 & & \\
\hline & Bolting & 3549009 & 2574520 & 2189878 & 72.54 & 61.70 & & \\
\hline & Flowering & 3628344 & 2484967 & 2092015 & 68.49 & 57.66 & & \\
\hline
\end{tabular}

\section{Functional annotation and pathway analysis of DEGs}

The cellular component, molecular function, and biological process associated with each of the DEGs were obtained using the Gene Ontology (GO) database. For example, there were more genes related to antioxidant activity, translation regulator activity, and reproduction in NHCC001 than in NHCC002 at the seedling stage. However, there were more auxiliary transport genes in NHCC002 than in NHCC001 (Additional file 2: Figure S6). The genes that belonged to the same orthologous cluster were classified into one group, based on the Clusters of Orthologous Groups (COG) database. Taking the DEGs of NHCC001 and NHCC002 at the seedling stage as an example, the cluster results showed that most DEG genes belonged to the general function category, followed by genes related to translation, ribosomal structure, and biogenesis (Additional file 2: Figure S7). Kyoto Encyclopedia of Genes and Genomes (KEGG) pathway analysis was performed to elucidate the energy metabolism, signal transduction, and biological systems of DEGs. We found that several DEGs are involved in several important pathways for plant growth and development, such as flowering genes and chlorophyll gene pathways, described in detail below.

\section{Analysis of flowering time genes}

We identified nearly 150 genes that had a tendency to increase from the adult to the bolting stages, corresponding

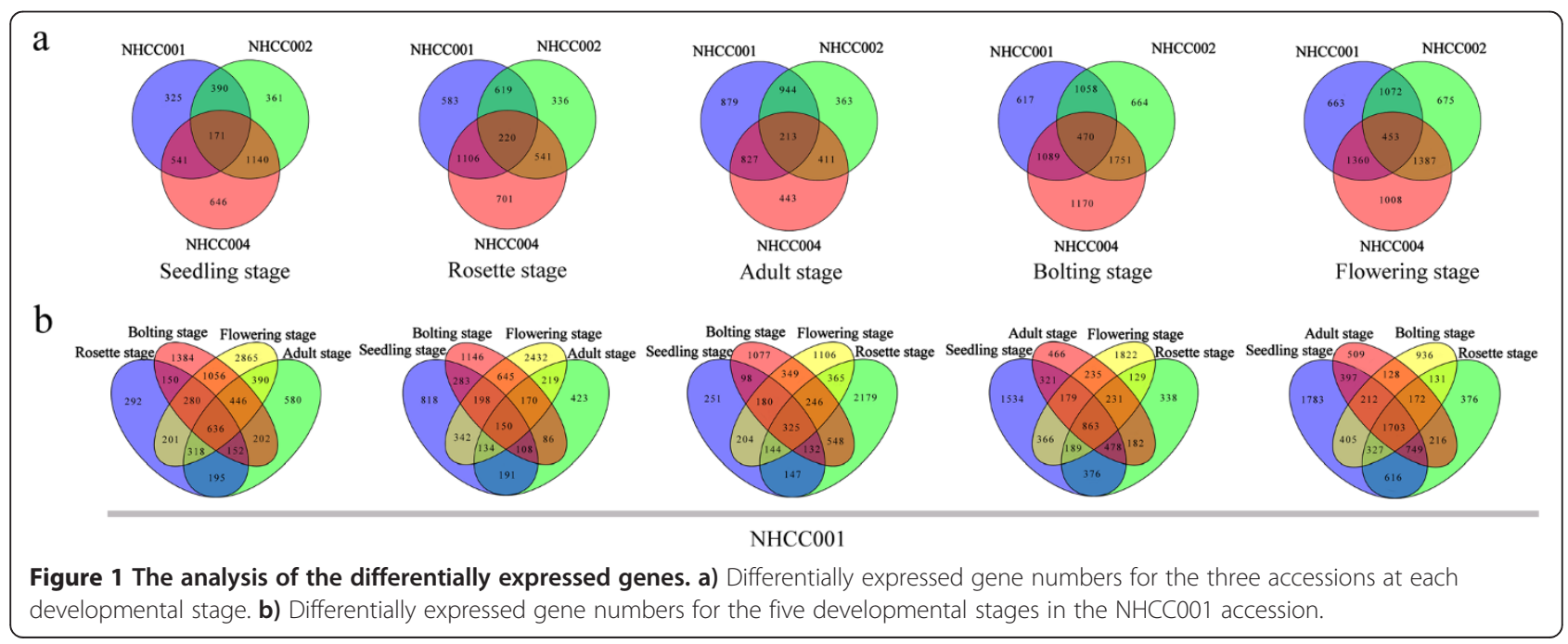




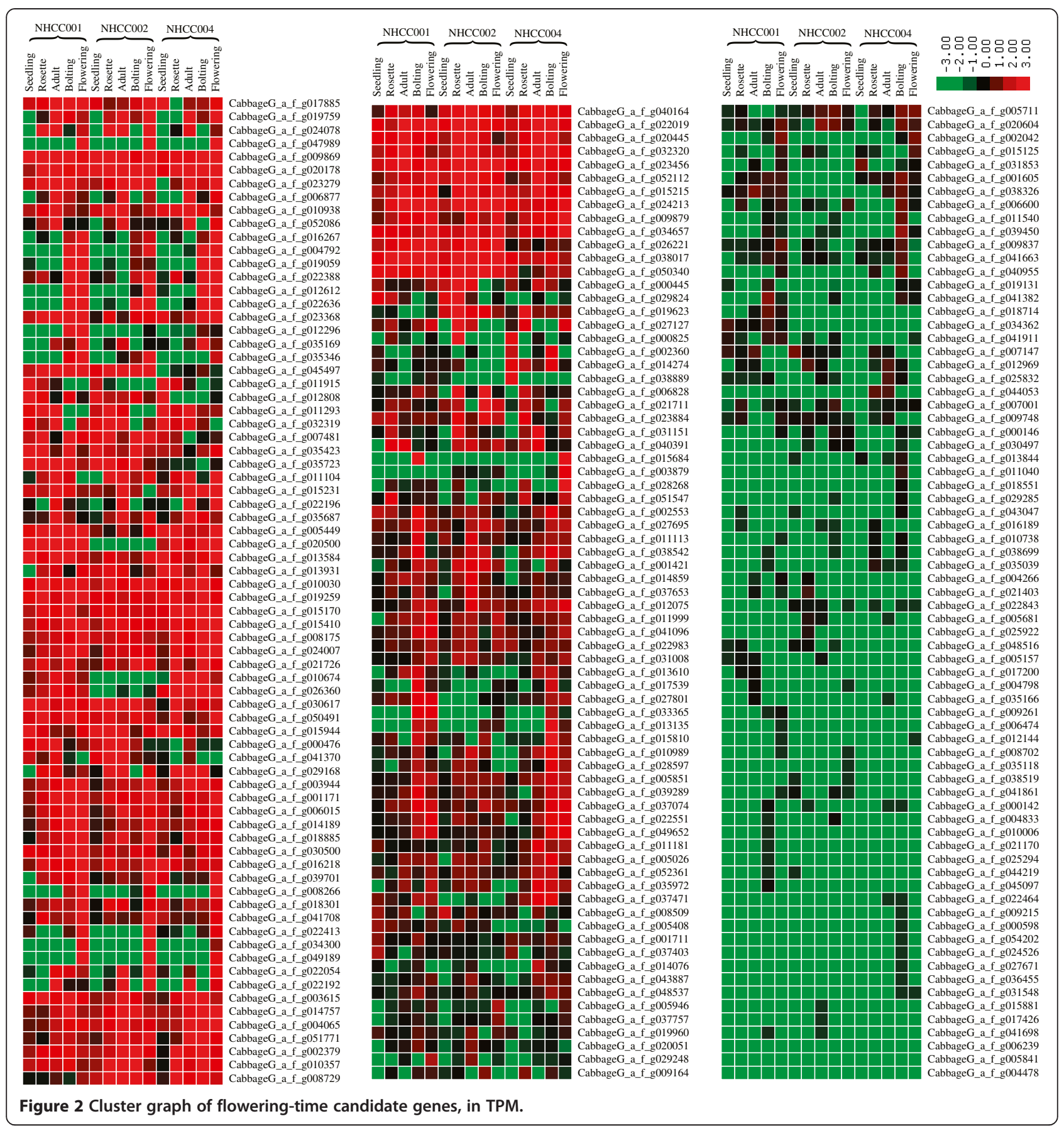

to the change from vegetative to reproductive growth. These genes are mainly involved in: transcription regulation pathways, such as for RNA-binding proteins; protein biosynthesis, such as ribosomal protein L1/L13/S7; ubiquitin signaling, such as ubiquitin protein, zinc finger (C3HC4-type RING finger), serine/threonine protein kinase, and glycine-rich protein GRP-3; and flower morphogenesis (MADS-box). In contrast, nearly 220 genes showed a tendency to decrease from the adult to the bolting stages. These genes are mainly involved in glutamine metabolism (such as glycosyltransferase family 14 [GT14] and sugar phosphate permease); the protein phosphatase pathway (such as serine/threonine protein kinase and serine/threonine protein phosphatase); transcription regulation (such as RNA-binding proteins, meprin, and TRAF homology domain-containing protein); protein biosynthesis (such as ribosomal protein L1/L10/L2/L4/L5/S10/L21E/ S12/S3AE and zinc finger protein); and some transcription factors (such as MAF1 [MADS AFFECTING FLOWERING 1], CBF2 [C-REPEAT/DRE BINDING FACTOR 2] 
and TINY [a member of the DREB subfamily A-4 of ERF/ AP2 transcription factor family].

MAF1 was considered a potential flowering inhibitor because it was specifically expressed in the vegetative stages (leaf, rosette, and adult). This result is consistent with Ratcliffe et al. [16] and He et al. [17]. CBF2 and TINY were expressed rarely in the bolting stage and not expressed in the flowering stage.

Seo et al. [18] found that overexpression of coldinducible $C B F \mathrm{~s}$ could increase expression of FLOWERING LOCUS C ( $F L C)$, an upstream negative regulator of $S O C 1$, thus delaying flowering. In addition, low temperature could induce the expression of the CBFs [19]. Overexpression of the $C B F 2$ gene also leads to increased freezing tolerance in Arabidopsis [20,21]. Because there is crosstalk between cold response and flowering, we hypothesized that decreased expression of $C B F 2$ was related to the conversion from vegetative to reproductive growth.

We also found some genes that are specifically related to the late flowering of NHCC004. An example is FLM (FLOWERING LOCUS M; CabbageG_a_f g029765, homologous with Bra024350), a MADS-box transcription factor that is a negative regulator of flowering [22] expressed in the bolting and flowering stages of NHCC004, but not in NHCC001 or NHCC002 (Additional file 2: Figure S8a). The results of qRT-PCR were in accord with this expression trend (Additional file 2: Figure $\mathrm{S} 8 \mathrm{~b}$ ). This suggests that FLM may be the reason for late flowering in NHCC004.

Gibberellin-insensitive gene (CabbageG_a_fg018551) was also found in the bolting stage of NHCC004, but not detected in any stages of NHCC001 or NHCC002 (Additional file 2: Figure S8c). The qRT-PCR results for this gene showed lower expression levels in NHCC001 and $\mathrm{NHCCOO2}$ relative to that of $\mathrm{NHCCOO4}$ at the bolting stage (Additional file 2: Figure S8d). Many studies have shown that gibberellin is required for flowering in Arabidopsis during short days [23,24]. Increased expression of gibberellin-insensitive gene in $\mathrm{NHCC004}$ weakens the role of gibberellin in promoting flowering. Therefore, high expression of flowering suppressor genes may be a reason for late flowering in NHCC004.

The FT (FLOWERING LOCUS T) gene (CabbageG_a_fg035346, homologous with the Bra004117, respectively), which promote flowering [25,26], were expressed only at the flowering stage in NHCC004, while they were expressed in the bolting and flowering stages in NHCCO01 and NHCCOO2 (Figure 3). In the circadian pathway of flowering, $F T$ was negatively regulated by ELF3 [27]. Our expression profiling analysis found that the ELF3 gene (CabbageG_a_f $g 020445)$ gradually increased and then decreased in all three accessions. The expression of ELF3 peaked during the adult stage of $\mathrm{NHCCOO1}$ and NHCC002 and in the bolting stage of NHCC004. The homologous genes, ELF4 (CabbageG_a_f_g013931) and
ELF6 (CabbageG_a_f_g052536), showed the same trend (Additional file 1: Table S2). These results suggest that delayed expression of flowering genes might further explain late flowering in $\mathrm{NHCCOO4.}$

Specific genes at the flowering stage may be related to the process of flower development and pollination. Our findings indicate that the expression levels of some genes, such as CabbageG_a_f_g015439 and CabbageG_ $a \_f g 018658$, were significantly highest at the flowering stage of all three accessions. CabbageG_a_f $g 015439$ gene, which encodes for ARK3 protein and is homologous to SLG (S locus glycoprotein), is involved in recognition of self-pollen [28,29]. CabbageG_a_f_g018658 gene encodes for AGL6 (agamous-like MADS-box protein 6) and functions as a DNA binding and transcription factor. Members of the MADS-box gene family have important roles in flower development, and participate in determining the identity of floral meristems early in flower development and of floral organ primordia later in flower development [30].

\section{Analysis of cold-tolerance genes}

From expression profiling, we found that two coldregulated genes (CabbageG_a_f_g014059 and CabbageG_a_f_g014057, homologous with Bra000265 and Bra000263, respectively, of heading Chinese cabbage) showed a higher expression level at the rosette stage (transcripts per million $[\mathrm{TPM}]>4000$ ) than at the other four stages (Figure 4).

Weather temperatures fluctuate significantly in Nanjing during autumn and winter. For example, in 2009 the temperature dropped from $10^{\circ} \mathrm{C}$ to $0^{\circ} \mathrm{C}$ over five days (from October 15 to October 20). These dramatic changes in the external temperature may be the reason for the high expression of cold-regulating genes. To test this inference, we studied the expression levels of these two genes using quantitative real-time PCR (Figure 5). The results showed that the relative expression values were $>1000$ after $12 \mathrm{~h}$ at $4^{\circ} \mathrm{C}$ treatment. In addition, the relative expression levels were also changed after abscisic acid (ABA) and polyethylene glycol (PEG) treatments. In general, low temperature, PEG, and ABA crosstalk to activate stress gene expression, and the expression of most cold-related genes is also affected by PEG or ABA treatments. Therefore, we suggest that the high expression of these genes was closely linked to cold resistance in non-heading Chinese cabbage.

\section{Analysis of self-incompatibility genes}

Three genes, CabbageG_a_f_g006792, CabbageG_a_f $g$ 011856, and CabbageG_a_fg039867, were expressed at all five stages and specifically existed only in NHCC002 (Additional file 2: Figure S9). These genes encode serine/threonine protein kinase, NAC domain-containing protein 82 , and dynein light chain type 1 family protein, respectively. 


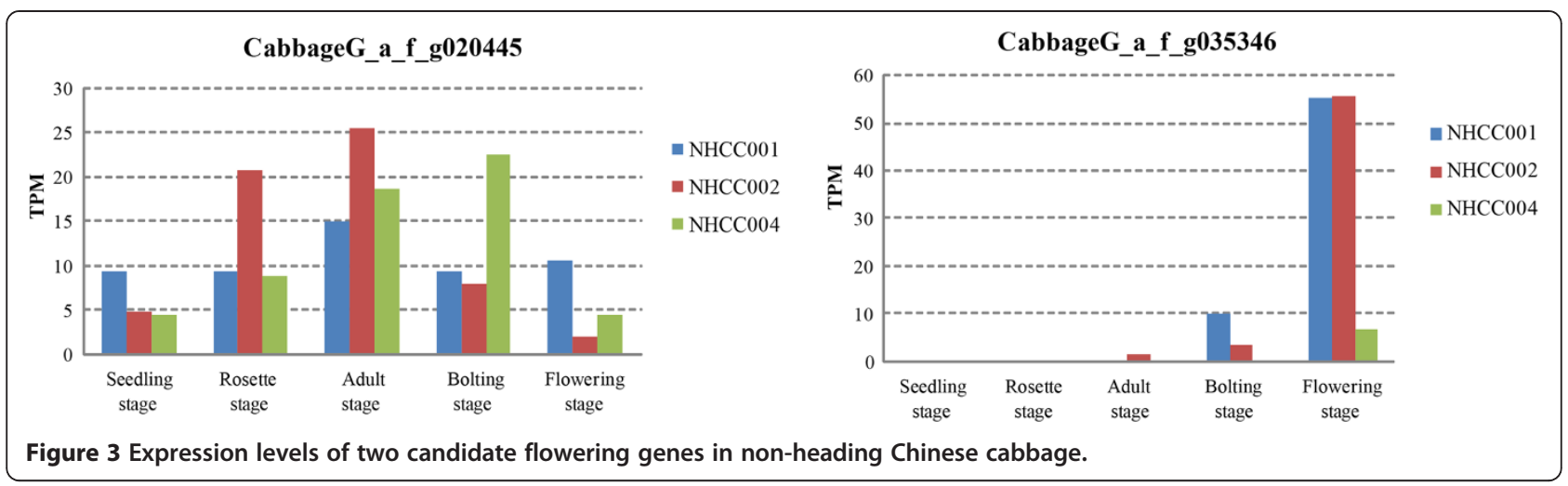

Among them, CabbageG_a_fg006792 and CabbageG_a_f $g 011856$ showed significantly decreased levels at the bolting and flowering stages compared with vegetative stages. However, the CabbageG_a_f_g039867 transcript increased at the bolting stage and declined at the flowering stage. In the process of self-pollination, serine/threonine protein kinases, such as S-locus receptor kinase (SRK) and M-locus protein kinase (MLPK), are involved in recognition and autophosphorylation in self-incompatible signaling pathways [31-33]. Thus, changes in the expression levels of CabbageG_a_f_g006792 could influence the activation of serine/threonine protein kinase, thereby affecting the recognition and rejection of self-pollen. NAC domain-containing proteins are plant-specific transcriptional factors involved in regulating several plant developmental processes, such as flower and embryo development [34-36]. Thus, these specifically expressed genes might be related to selfincompatibility of NHCCOO2.

We also found that CabbageG_a_f_g031080 had higher transcripts at all stages in accessions NHCC001 and
NHCC004, while it was expressed at low levels at the bolting and flowering stages in NHCC002 (Additional file 2: Figure S9). This gene encodes ribosomal protein L13, which is involved in the assembly of proteins. The downregulation of ribosomal protein L13 might be related to the suppression of self-pollen development in NHCC002. Compared with NHCCOO1 and NHCC004, 46 genes of the NHCC002 accession showed lower transcript levels, with zero TPM, even during flowering.

Interestingly, some genes encoded vesicle coat complex and ATEXO70H7, which are related to secretory protein trafficking and polarized exocytosis [37,38]. Exo70A1 participates in the growth of pollen tube tips and has been identified as a negative regulator in the Brassica selfincompatibility response $[39,40]$. We found that the expression levels of vesicle coat complex and ATEXO70H7 were higher in NHCCOO1 and NHCCOO4 compared with NHCC002 accession, given that the expression levels of these proteins were nil at the flowering stage. The low abundance of vesicle coat complex and ATEXO70H7
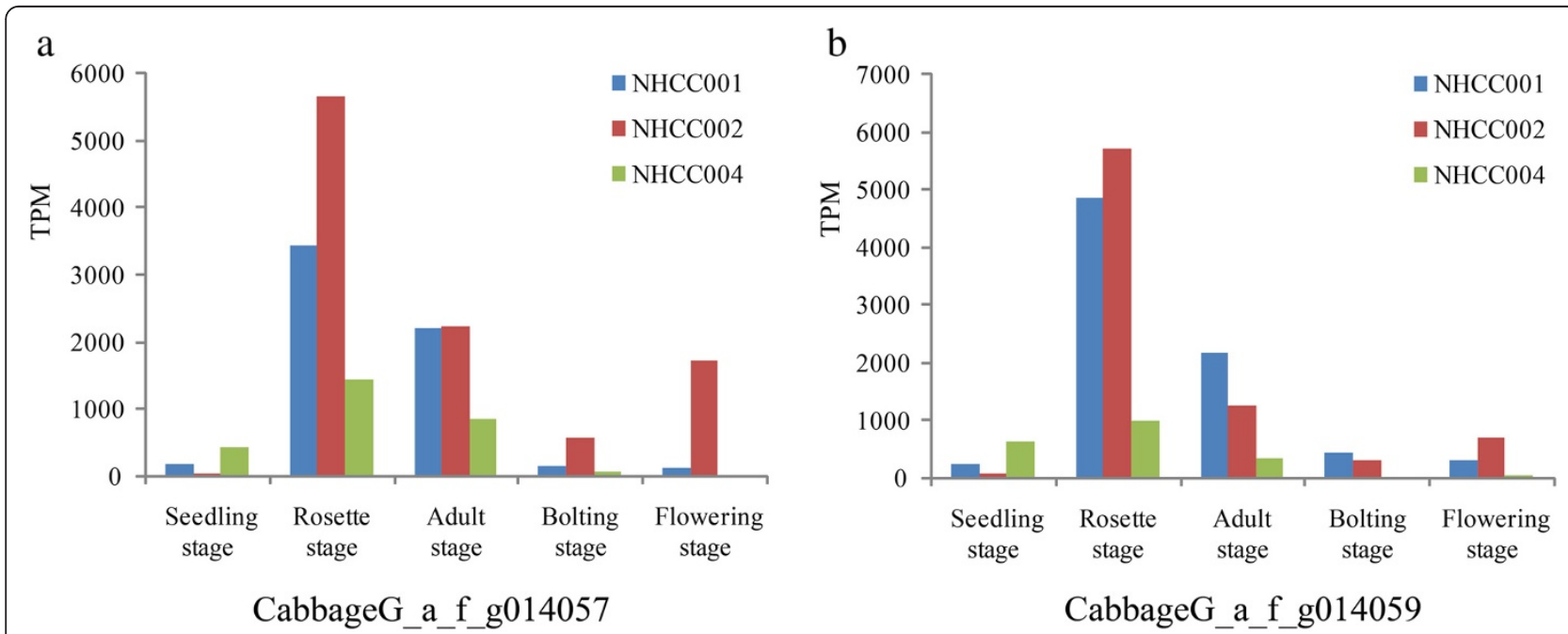

Figure 4 Cold-tolerance genes identified from differentially expressed genes in non-heading Chinese cabbage. a) CabbageG_a_f_g014057; b) CabbageG_a_f_g014059. 


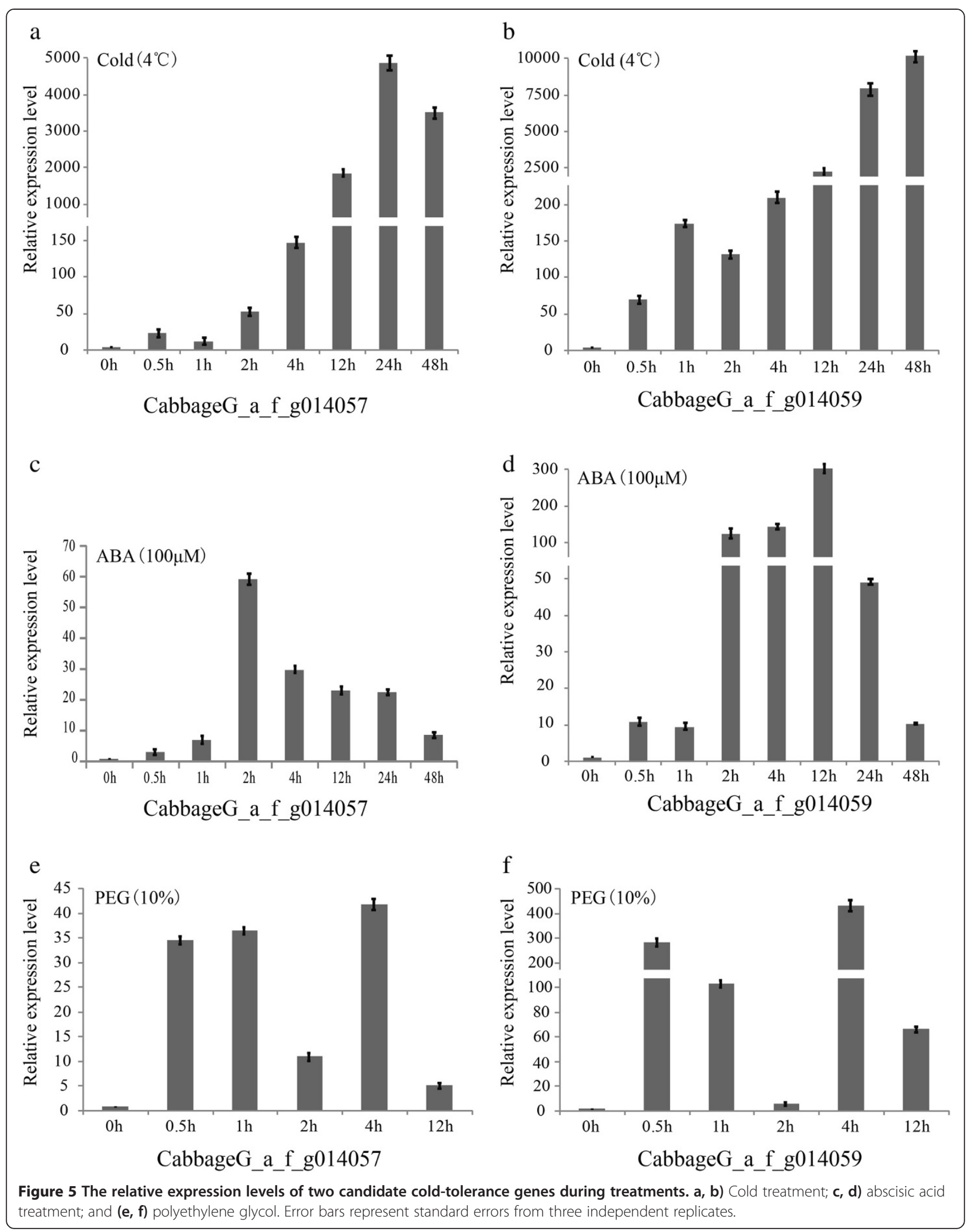


implied that they might have similar functions as negative regulators in the self-incompatibility response of NHCC002.

\section{Analysis of leaf color genes}

We analyzed the genes associated with leaf color. These genes have an important role in the control of chlorophyll biosynthesis, chloroplast structure, and plant development. Moreover, they might affect crop yields by regulating photosynthesis. Therefore, it is crucial for improving crop production to identify leaf-color related genes and uncover the genetic basis of the leaf color trait. The chlorophyll content of the leaves was measured using a portable chlorophyll meter (SPAD-502Plus, Konica Minolta). The measurement results showed that the chlorophyll indices of $\mathrm{NHCCOO1}$ and $\mathrm{NHCCOO2}$ were significantly different at the rosette stage. After analyzing the genes involved in the chlorophyll gene (KO00860) pathway of these two accessions, we found that the light leaf color of NHCC002 is most likely due to decreased chlorophyll synthesis, perhaps resulting from lower chlorophyllase activity $[41,42]$.

The CabbageG_a_f_g026085 gene, which encodes the chloroplastic protein FLUORESCENT IN BLUE LIGHT, was also expressed at lower levels in NHCC002. Furthermore, qRT-PCR showed the same expression pattern as the expression profile (Additional file 2: Figure S10). It is involved in the regulation of chlorophyll biosynthesis and might be a negative regulator of tetrapyrrole biosynthesis in chloroplasts [43,44]. This result implied that CabbageG_a $\_\_g 026085$ could also be a candidate gene related to chlorophyll biosynthesis in non-heading Chinese cabbage.

\section{DEGs in the genomic colinear blocks between B. rapa and A. thaliana}

In our expression profile, 29101 genes were expressed and 28638 (98.4\%) of the expressed genes were mapped to 10 chromosomes. In these expressed genes, 15830 (54.4\%) were identified as DEGs of different accessions or developmental stages, and 15567 (98.3\%) genes were located on the 10 chromosomes. To show the up- and downregulation of DEGs in a more intuitive way, we labeled the DEGs on each chromosome with the regulation information (Figure 6, Additional file 2: Figure S11).

A total of 581 colinear blocks were identified between the genomes of non-heading Chinese cabbage and Arabidopsis. Finally, 369 (63.5\%) colinear blocks were obtained after removing the blocks that contained fewer than 10 genes from consideration. Of the 15830 DEGs, nearly half of them $(7504,47.4 \%)$ were located in the colinear blocks (Figure 7). In non-heading Chinese cabbage and heading Chinese cabbage, 710 colinear blocks were identified. Four hundred and twelve (58.0\%) colinear blocks were obtained after removing the blocks containing fewer than 10 genes.
A total of 23.1\% (3652) of the DEGs were identified in the colinear blocks (Additional file 2: Figure S12).

To further characterize the relationships among nonheading Chinese cabbage, heading Chinese cabbage, and Arabidopsis we analyzed the paralogous and orthologous genes among them. There were 31 322, 20 770, and 23 171 paralogous gene pairs in the entire genomes of nonheading Chinese cabbage, heading Chinese cabbage, and Arabidopsis, respectively. For the orthologous genes, there were 46716 gene pairs between non-heading Chinese cabbage and Arabidopsis, whereas there were 64975 gene pairs between non-heading Chinese cabbage and heading Chinese cabbage. Furthermore, we investigated the number of differentially expressed paralogous genes that existed in non-heading Chinese cabbage, and we found that $4092(25.8 \%)$ genes had a paralogous gene in nonheading Chinese cabbage (Additional file 1: Table S6). Of these genes, 1,960 had only one paralog. The number of the paralogs was $>10$ for 117 DEGs, and $>50$ for 16 DEGs.

Most of the genes that had $>50$ paralogs belonged to the non-long terminal repeat retroelement reverse transcriptase. We used the Pfam program (http://pfam.sanger.ac.uk/) [45] to identify 3840 paralogs that belonged to 9183 DEGs. Among these paralogs, most encoded proteins were leucinerich repeat protein, protein kinase domain protein, WD domain, and G-beta repeat (Additional file 2: Figure S13).

We also analyzed orthologous pairs of DEGs, identifying 13932 genes that were orthologous between the nonheading Chinese cabbage and heading Chinese cabbage. Of these, 9012 genes had one ortholog in heading Chinese cabbage, 314 genes had $>10$ orthologs in heading Chinese cabbage, and one gene (CabbageG_a_f_g017292) had 54 orthologs in heading Chinese cabbage. While there was a total of 11512 orthologs (72.7\%) between non-heading Chinese cabbage and Arabidopsis (Additional file 1: Table S7), 7368 genes had only one ortholog in Arabidopsis, which decreased to 199 when the number of orthologs was $>10$. Interestingly, the same gene (CabbageG_a f_g017292) also had a relatively high number of orthologs (i.e., 56) in Arabidopsis. The same gene also had 37 paralogs in non-heading Chinese cabbage. Although the explanation for the high number of copy number variations for this gene is unknown, we inferred that it might affect plant growth. Therefore, we annotated its function, which revealed that it was a disease resistance protein (TIR-NBS-LRR class).

\section{Conclusions}

In our analysis, we identified numerous DEGs related to important agricultural traits. By comparing cultivars and developmental stages, we found many genes associated with flowering time, self-incompatibility, cold-tolerance, and leaf color. Although the functions of most of the other DEGs are not known, this study will further our understanding of the expression pattern of these genes 


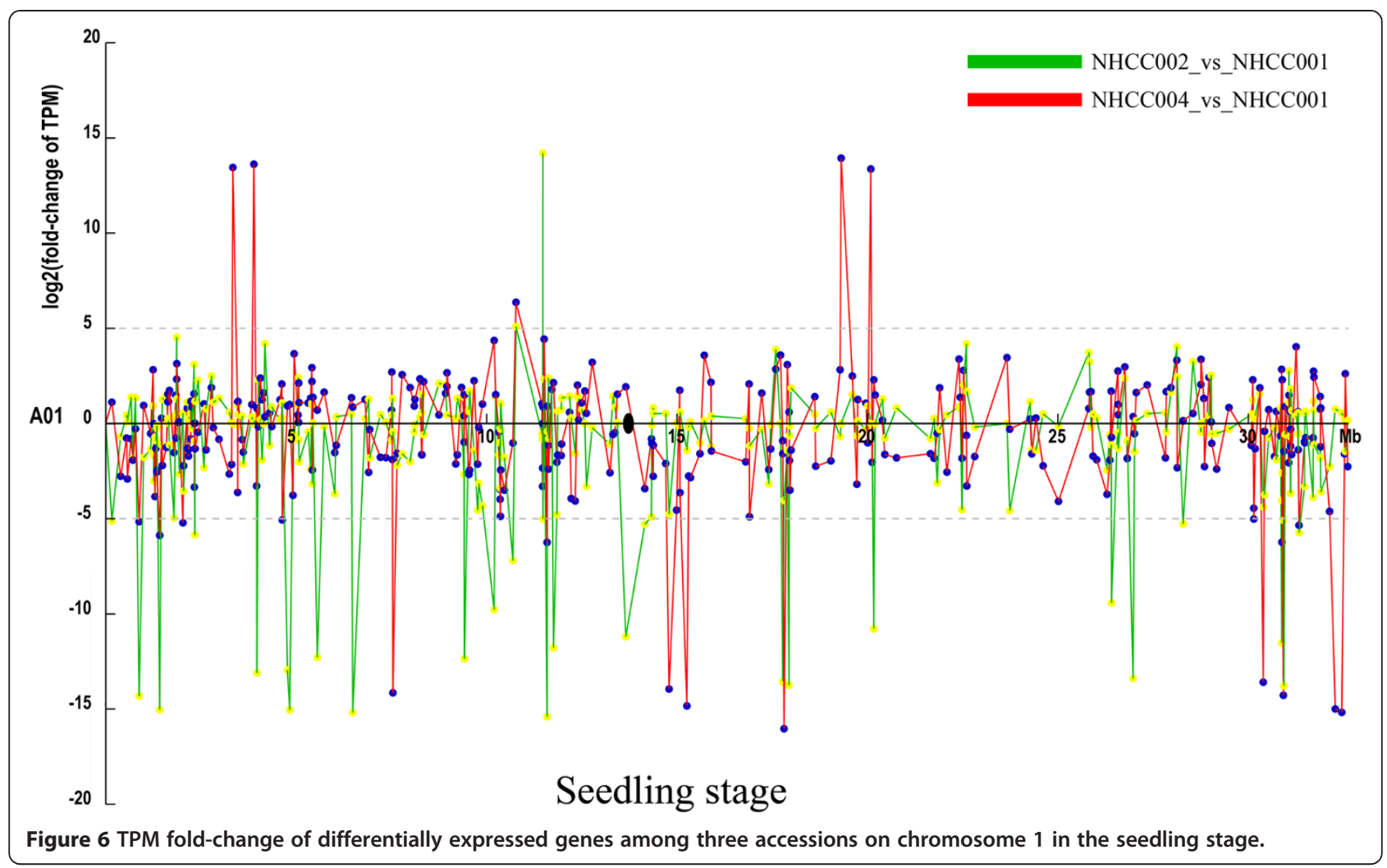

and genetic improvement of non-heading Chinese cabbage or other cruciferous vegetables, as well as basic biological research. In particular, clarification of the regulatory networks involved in flowering will contribute to the cultivation of new late-flowering varieties, which can provide a wealth of resources for breeding. This detailed analysis of the expression profiles of non-heading Chinese cabbage provides the first comprehensive review of the expression patterns of five development stages, and has unveiled numerous candidate genes that may underlie morphological and genetic polymorphisms of non-heading Chinese cabbage.

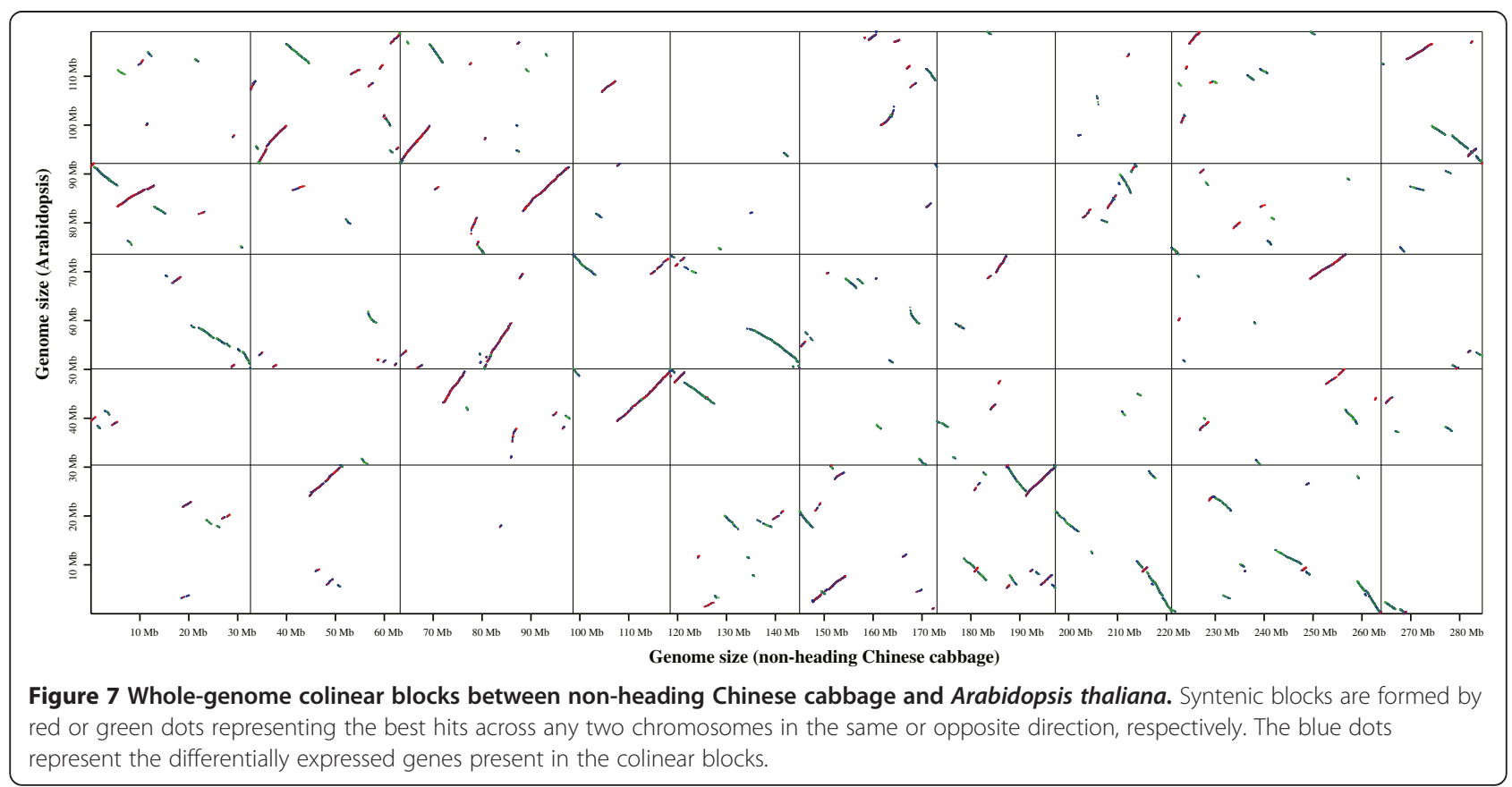




\section{Materials and methods Sample preparation}

The non-heading Chinese cabbage accessions NHCC001, $\mathrm{NHCCO02}$, and NHCC004 used for expression profiles were cultivated in the field under a non-controlled environment. RNA was extracted at the five important plant developmental stages: seedling, rosette, adult, bolting, and flowering. During the first three stages, RNA for expression profiles was extracted from leaves. The RNA of the bolting stage was extracted from an equal mixture of leaf and buds, while leaves and flowers were used for RNA extractions during flowering. All the RNA was extracted in accordance with the manufacturer's instructions for the RNeasy plant mini kit (Qiagen).

\section{Digital gene expression tag profiling}

The expression levels of genes for the three accessions at the five development stages were obtained using digital gene expression-tag profiling methods, as in a previous report [15]. The number of times that a unique tag sequence was detected represents the quantitative expression of the corresponding transcript in tissues.

The Bowtie program (http://bowtie-bio.sourceforge.net/ index.shtml) was used to map sequencing reads to the non-heading Chinese cabbage genome [46]. Finally, high quality clean tags were compared with the genome sequences of non-heading Chinese cabbage and the expression level of each gene was quantified as TPM [47].

To evaluate the mRNA expression characteristics, we analyzed the TPM of each identified gene. The highly expressed genes (defined as TPM $>100$ ) accounted for $>50 \%$ of the total expression tags from all genes (Additional file 2: Figure S14a) in the NHCC001 accession. The number of genes with a TPM $<10$ was $\sim 55 \%$ of all genes, while the number of genes with a TPM $>80$ only accounted for $\sim 10 \%$ of all genes (Additional file 2: Figure S14b). Statistical analysis of the other two accessions showed similar patterns, illustrating that most genes were expressed at a low level, and only a handful of genes expressed at high levels accounted for the majority of tag reads. This is in accord with the heterogeneous and redundant features of mRNA expression.

We also performed saturation analyses of sequencing data. The data show that the more sequencing tags that a gene had, the more likely it was to be expressed in a certain range. When the number of tags reached a threshold, the number of expressed genes approached saturation. Our analysis shows that the number of expressed genes was nearly saturated when tag number was $>3$ million (Additional file 2: Figure S14c). The lowest number of tags obtained in our study was 3.40 million for the rosette stage of NHCC004. Therefore, our sequencing reads had reached saturation for all the sample stages, assuring that most of the expressed genes during plant growth and development were detected in our study.

\section{Identification of DEGs}

The DEGs were identified mainly as previously described [48]. The expressed genes were identified using IDEG6 software [49], and a general chi-squared test was used to test the hypothesis. The false discovery rate was used to correct the $P$-value [50], and the fold changes were also calculated for identification of the differentially expressed genes. To avoid the potential noise signal from highthroughput sequencing, absolute fold change $\geq 2.0$ and a false discovery rate $<0.01$ were used to define the DEGs, including the upregulated and downregulated genes. The differential expression levels of the genes at the five stages of the three accessions were compared and visualized through scatter plots drawn by Perl scripts, and their expression pattern was displayed using the heatmap function in the Cluster program (http://bonsai.hgc.jp/ mdehoon/ software/cluster/software.htm) and visualized using Tree View software (http://jtreeview.sourceforge.net/) [51]. An interaction network of the DEGs was constructed using Cytoscape software (http://www.cytoscape.org/) according to the expression level of the genes [52]. The numbers of specific and common DEGs were plotted using the Venn diagram function in the $\mathrm{R}$ software package [53].

\section{Plant materials, growth conditions, and stress treatments}

To verify the two candidate cold-related genes, the nonheading Chinese cabbage cultivar 'Suzhouqing' (NHCC001) was used for quantitative real-time PCR. Seeds were grown in pots containing a soil: vermiculite (3:1) mixture in a controlled-environment growth chamber programmed for $16 / 8 \mathrm{~h}$ at $25 / 20^{\circ} \mathrm{C}$ for day/night, relative humidity of 55 $60 \%$. At the rosette stage, they were transferred to growth chambers set at $4^{\circ} \mathrm{C}$ under the same light intensity and day length as the cold treatments. The leaf samples were collected at $0,0.5,1,2,4,12,24$, and $48 \mathrm{~h}$ after cold treatment. At the same time for acclimation, some plants were cultured in $1 / 2$ Hoagland's solution in plastic containers, with $\mathrm{pH}$ at 6.5. After 5 days of acclimatization, plants were cultured in $100 \mu \mathrm{M}$ abscisic acid, $10 \%$ polyethylene glycol, or left untreated. Leaf samples were collected $0,0.5,1,2,4,12$, 24 , and $48 \mathrm{~h}$ after these treatments and then frozen in liquid nitrogen and stored at $-70^{\circ} \mathrm{C}$ until further analysis.

\section{RNA isolation and quantitative real-time PCR analysis}

Total RNA was isolated from leaves using an RNA kit (Tiangen, China) in accordance with the manufacturer's instructions. The RNA was reverse transcribed into cDNA using the Prime Script RT reagent kit (TaKaRa, Japan). The actin gene (AF111812) was used as an internal control to normalize the expression level of the target gene among different samples [54]. The specific primers were designed according to the target gene sequences by Primer 5.0 software (Additional file 1: Table S8). Quantitative real-time PCR assays were performed with three biological and 
three technical replicates. Each reaction was performed in a $20-\mu \mathrm{L}$ reaction mixture containing diluted cDNA as template, SYBR Premix Ex Taq $(2 \times)$ (TaKaRa, Japan), and gene-specific primers. Quantitative real-time PCR was performed using MyiQ Single-Color Real-Time PCR Detection System (Bio-rad, Hercules, CA) with the following cycling profile: $94^{\circ} \mathrm{C}$ for $30 \mathrm{~s}$, and then 40 cycles at $94^{\circ} \mathrm{C}$ for $10 \mathrm{~s}, 58^{\circ} \mathrm{C}$ for $30 \mathrm{~s}$, and then a melting curve $(61$ cycles at $65^{\circ} \mathrm{C}$ for $10 \mathrm{~s}$ ) was generated to check the specific amplification. The relative quantitative method was employed to analyze the relative gene expression level. RNA levels were expressed relative to that of the actin gene (AF111812) as $2^{-\Delta \Delta C T}$, where $\mathrm{Ct}$ is the cycle threshold, in accordance with previous studies [55].

\section{Functional annotation and pathway analysis}

The annotations of DEGs in non-heading Chinese cabbage were obtained by searching the protein databases Iprscan (http://www.ebi.ac.uk/Tools/pfa/iprscan/), UniProtKB (http://www.ebi.ac.uk/uniprot/) [56], TrEMBL (http://www.ebi.ac.uk/uniprot/TrEMBLstats/) [57], GO (http://www.geneontology.org/) [58], and KEGG (http:// www.genome.jp/kegg/) [59] and the annotations obtained from these five protein databases were integrated using Perl script. In addition, the biological processes and functions of DEGs were analyzed using the COG (http://www. ncbi.nlm.nih.gov/COG/) [60], and GO databases. The COG database represents major phylogenetic lineages, and each COG consists of individual proteins or groups of paralogs from at least 3 lineages.

\section{Mapping differentially expressed genes on the draft genome}

The distribution of all predicted genes, expressed genes, and differentially expressed genes on chromosomes were visualized using Perl scripts, and differently colored lines represented each gene dataset. The orthologous and paralogous genes were identified using OrthoMCL software (http://www.orthomcl.org/cgi-bin/OrthoMclWeb.cgi) [61], and the copy number of these genes was calculated using Perl scripts. The syntenic relationships between species was constructed by McScan (MATCH_SCORE: 40, MATCH_SIZE: 5, GAP_SCORE:-2, EXTENSION_DIST: 40, E_VALUE: 1e-05; http://chibba.agtec.uga.edu/duplication/mcscan/) [62]. The all-against-all BLASTP comparison provided the E-value and the pairwise gene information for protein clustering. Paired segments were extended by identifying clusters of genes. This method was used to build the genome synteny blocks of non-heading Chinese cabbage compared with heading Chinese cabbage and Arabidopsis. Furthermore, we filtered the synteny blocks that had $<10$ genes to obtain improved collinear analysis. Finally, the DEGs that were located in the synteny blocks were marked according to the physical position, and the collinear blocks plus the marked DEGs were plotted using Perl scripts.

\section{Additional files}

\begin{abstract}
Additional file 1: Table S1. Expression profile data at five development stages of three non-heading Chinese cabbage accessions. Table S2. Expression values at five development stages of three non-heading Chinese cabbage (TPM). Table S3. The number of differentially expressed genes among different evelopmental stages for each accession. Table S4. Differentially expressed genes among three accessions at the same developmental stage. Table S5. Statistical analysis of the differentially expressed genes between NHCCOO1 and $\mathrm{NHCCO} 2$ at the seedling stage. Table S6. Number of paralogous genes in differentially expressed genes of non-heading Chinese cabbage. Table S7. Number of orthologous genes in differentially expressed genes of non-heading Chinese cabbage compared with Arabidopsis and heading Chinese cabbage. Table S8. Primer sequences used for quantitative real-time PCR amplification of actin and two cold-tolerance genes.

Additional file 2: Figure S1. Number of expressed genes in each

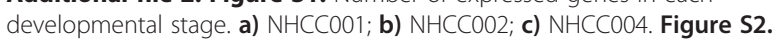
Numbers of expressed genes for the three accessions at each developmental stage. a) Seedling; b) rosette; c) adult; d) bolting; e) flowering. Figure S3. Numbers of differentially expressed genes for the five developmental stages in accessions NHCC002 and NHCCO04. Figure S4. Genes differentially upregulated or downregulated in the seedling stage among the accessions. The red dots represent the upregulated genes, and the green dots represent the downregulated genes. Figure S5. Cluster graph of self-incompatibility candidate genes, in TPM. Figure S6. GO annotation of the differentially expressed genes. Figure S7. COG of the differentially expressed genes. Figure S8. Expression levels of two candidate flowering genes. a, c) Transcription per million, by expression profile; and $\mathbf{( b ,} \mathbf{d})$ relative expression levels, by qRT-PCR.

Figure S9. Self-incompatibility genes identified from differentially expressed genes in non-heading Chinese cabbage. Figure S10. The expression levels and pathways of chlorophyll genes involved in non-heading Chinese cabbage. Figure S11. TPM fold-changes of differentially expressed genes among the three accessions at each stage on chromosome. (l.e., on chromosome 1 for the rosette, adult, bolting, and flowering stages.). Figure S12. Whole-genome colinear blocks between non-heading Chinese cabbage and heading Chinese cabbage. Syntenic blocks are formed by red or green dots representing the best hits across any two chromosomes in the same or opposite directions, respectively. The blue dots represent the differentially expressed genes present in the colinear blocks. Figure S13. Pfam domain annotation for the differentially expressed genes belonging to the paralogous genes and other differentially expressed genes. Figure S14. Statistical analysis of expression profile data. a) Distribution charts of TPM; b) statistical charts for the gene numbers in each TPM interval region; c) sequencing saturation analysis chart.
\end{abstract}

\section{Competing interests}

The authors declare that they have no competing interests.

\section{Authors' contributions}

The study was conceived by XS and XH. XH and XS collected the dataset of non-heading Chinese cabbage. XS contributed to data analysis, bioinformatics analysis, and manuscript preparation. XS and YL participated in writing the manuscript. All authors contributed to revising the manuscript. All authors read and approved the final manuscript.

\section{Acknowledgements}

This work was supported by the National Program on Key Basic Research Projects (The 973 Program: 2012CB113900), National Natural Science Foundation of China (Key Program, No.31330067), and National High Technology Research and Development Program of China (863 Program, No. 2012AA100101).

Received: 23 October 2013 Accepted: 18 March 2014

Published: 22 March 2014 


\section{References}

1. Koornneef M, Alonso-Blanco C, Blankestijn-de Vries H, Hanhart CJ, Peeters AJM: Genetic interactions among late-flowering mutants of Arabidopsis. Genetics 1998, 148(2):885-892.

2. Araki T, Kobayashi Y, Kaya H, Iwabuchi M: The flowering-time gene FT and regulation of flowering in Arabidopsis. J Plant Res 1998, 111(1102):277-281.

3. Putterill J, Laurie R, Macknight R: It's time to flower: the genetic control of flowering time. Bioessays 2004, 26(4):363-373.

4. Onouchi H, Igeno Ml, Perilleux C, Graves K, Coupland G: Mutagenesis of plants overexpressing CONSTANS demonstrates novel interactions among Arabidopsis flowering-time genes. Plant Cell 2000, 12(6):885-900.

5. Samach A, Onouchi H, Gold SE, Ditta GS, Schwarz-Sommer Z, Yanofsky MF, Coupland G: Distinct roles of CONSTANS target genes in reproductive development of Arabidopsis. Science 2000, 288(5471):1613-1616.

6. Wang ZJ, Huang JQ, Huang YJ, Chen FF, Zheng BS: Cloning and Characterization of a Homologue of the FLORICAULA/LEAFY Gene in Hickory (Carya cathayensis Sarg). Plant Mol Biol Rep 2012, 30(3):794-805.

7. Mouradov A, Cremer F, Coupland G: Control of flowering time: Interacting pathways as a basis for diversity. Plant Cell 2002, 14:S111-S130.

8. Kim SY, Park BS, Kwon SJ, Kim J, Lim MH, Park YD, Kim DY, Suh SC, Jin YM, Ahn JH, Lee YH: Delayed flowering time in Arabidopsis and Brassica rapa by the overexpression of FLOWERING LOCUS C (FLC) homologs isolated from Chinese cabbage (Brassica rapa L. ssp pekinensis). Plant Cell Rep 2007, 26(3):327-336.

9. Charlesworth D, Vekemans X, Castric V, Glemin S: Plant self-incompatibility systems: a molecular evolutionary perspective. New Phytol 2005, 168(1):61-69.

10. Busch JW, Schoen DJ: The evolution of self-incompatibility when mates are limiting. Trends Plant Sci 2008, 13(3):128-136.

11. Vekemans X, Slatkin M: Gene and Allelic Genealogies at a Gametophytic Self-Incompatibility Locus. Genetics 1994, 137(4):1157-1165.

12. Muirhead CA: Consequences of population structure on genes under balancing selection. Evolution 2001, 55(8):1532-1541.

13. Goubet PM, Berges H, Bellec A, Prat E, Helmstetter N, Mangenot S, Gallina S, Holl AC, Fobis-Loisy I, Vekemans $X$, Castric V: Contrasted patterns of molecular evolution in dominant and recessive self-incompatibility haplotypes in Arabidopsis. PLoS Genet 2012, 8(3):e1002495.

14. Feng L, Liu H, Liu Y, Lu Z, Guo G, Guo S, Zheng H, Gao Y, Cheng S, Wang J, Zhang $\mathrm{K}$, Zhang $Y$ : Power of deep sequencing and agilent microarray for gene expression profiling study. Mol Biotechnol 2010, 45(2):101-110.

15. Wang Z, Dong D, Ru B, Young RL, Han N, Guo T, Zhang S: Digital gene expression tag profiling of bat digits provides robust candidates contributing to wing formation. BMC Genomics 2010, 11:619.

16. Ratcliffe OJ, Nadzan GC, Reuber TL, Riechmann JL: Regulation of flowering in Arabidopsis by an FLC homologue. Plant Physiol 2001, 126(1):122-132.

17. He C, Sommer H, Grosardt B, Huijser P, Saedler H: PFMAGO, a MAGO NASHI-like factor, interacts with the MADS-domain protein MPF2 from Physalis floridana. Mol Biol Evol 2007, 24(5):1229-1241.

18. Seo E, Lee H, Jeon J, Park H, Kim J, Noh YS, Lee I: Crosstalk between cold response and flowering in Arabidopsis is mediated through the flowering-time gene SOC1 and its upstream negative regulator FLC. Plant Cell 2009, 21(10):3185-3197.

19. Zarka DG, Vogel JT, Cook D, Thomashow MF: Cold induction of Arabidopsis CBF genes involves multiple ICE (inducer of CBF expression) promoter elements and a cold-regulatory circuit that is desensitized by low temperature. Plant Physiol 2003, 133(2):910-918.

20. Hu P, Maiti T: A nonparametric mean-variance smoothing method to assess Arabidopsis cold stress transcriptional regulator CBF2 overexpression microarray data. PLoS One 2011, 6(5):e19640.

21. Sharabi-Schwager M, Lers A, Samach A, Guy CL, Porat R: Overexpression of the CBF2 transcriptional activator in Arabidopsis delays leaf senescence and extends plant longevity. J Exp Bot 2010, 61(1):261-273.

22. Scortecci KC, Michaels SD, Amasino RM: Identification of a MADS-box gene, FLOWERING LOCUS M, that represses flowering. Plant J 2001, 26(2):229-236

23. Moon J, Suh SS, Lee H, Choi KR, Hong CB, Paek NC, Kim SG, Lee I: The SOC1 MADS-box gene integrates vernalization and gibberellin signals for flowering in Arabidopsis. Plant J 2003, 35(5):613-623.

24. Wilson RN, Heckman JW, Somerville CR: Gibberellin Is Required for Flowering in Arabidopsis thaliana under Short Days. Plant Physiol 1992, 100(1):403-408.
25. Lopez-Vernaza M, Yang S, Muller R, Thorpe F, de Leau E, Goodrich J: Antagonistic roles of SEPALLATA3, FT and FLC genes as targets of the polycomb group gene CURLY LEAF. PLoS One 2012, 7(2):e30715.

26. Kobayashi $Y$, Weigel $D$ : Move on up, it's time for change-mobile signals controlling photoperiod-dependent flowering. Genes Dev 2007, 21(19):2371-2384.

27. Kinoshita T, Ono N, Hayashi Y, Morimoto S, Nakamura S, Soda M, Kato Y, Ohnishi M, Nakano T, Inoue S, Shimazaki K: Flowering Locus T regulates stomatal opening. Curr Biol 2011, 21(14):1232-1238.

28. Scutt CP, Gates PJ, Gatehouse JA, Boulter D, Croy RR: A cDNA encoding an S-locus specific glycoprotein from Brassica oleracea plants containing the S5 self-incompatibility allele. Mol Gen Genet 1990, 220(3):409-413.

29. Delorme V, Giranton JL, Hatzfeld Y, Friry A, Heizmann P, Ariza MJ, Dumas C, Gaude T, Cock JM: Characterization of the S locus genes, SLG and SRK, of the Brassica S3 haplotype: identification of a membrane-localized protein encoded by the S locus receptor kinase gene. Plant J 1995, 7(3):429-440.

30. Rounsley SD, Ditta GS, Yanofsky MF: Diverse roles for MADS box genes in Arabidopsis development. Plant Cell 1995, 7(8):1259-1269.

31. Giranton JL, Dumas C, Cock JM, Gaude T: The integral membrane S-locus receptor kinase of Brassica has serine/threonine kinase activity in a membranous environment and spontaneously forms oligomers in planta. Proc Natl Acad Sci U S A 2000, 97(7):3759-3764.

32. Cabrillac D, Cock JM, Dumas C, Gaude T: The S-locus receptor kinase is inhibited by thioredoxins and activated by pollen coat proteins. Nature 2001, 410(6825):220-223.

33. Murase K, Shiba H, Iwano M, Che FS, Watanabe M, Isogai A, Takayama S: A membrane-anchored protein kinase involved in Brassica selfincompatibility signaling. Science 2004, 303(5663):1516-1519.

34. Sablowski RW, Meyerowitz EM: A homolog of NO APICAL MERISTEM is an immediate target of the floral homeotic genes APETALA3/PISTILLATA. Cell 1998, 92(1):93-103.

35. Duval M, Hsieh TF, Kim SY, Thomas TL: Molecular characterization of AtNAM: a member of the Arabidopsis NAC domain superfamily. Plant Mol Biol 2002, 50(2):237-248.

36. Nuruzzaman M, Manimekalai R, Sharoni AM, Satoh K, Kondoh H, Ooka H, Kikuchi S: Genome-wide analysis of NAC transcription factor family in rice. Gene 2010, 465(1-2):30-44.

37. Springer S, Schekman R: Nucleation of COPII vesicular coat complex by endoplasmic reticulum to Golgi vesicle SNAREs. Science 1998, 281(5377):698-700

38. Li S, van Os GM, Ren S, Yu D, Ketelaar T, Emons AM, Liu CM: Expression and functional analyses of EXO70 genes in Arabidopsis implicate their roles in regulating cell type-specific exocytosis. Plant Physiol 2010, 154(4):1819-1830.

39. Hala M, Cole R, Synek L, Drdova E, Pecenkova T, Nordheim A, Lamkemeyer T, Madlung J, Hochholdinger F, Fowler JE, Zarsky V: An exocyst complex functions in plant cell growth in Arabidopsis and tobacco. Plant Cell 2008, 20(5):1330-1345.

40. Samuel MA, Chong YT, Haasen KE, Aldea-Brydges MG, Stone SL, Goring DR Cellular pathways regulating responses to compatible and selfincompatible pollen in Brassica and Arabidopsis stigmas intersect at Exo70A1, a putative component of the exocyst complex. Plant Cell 2009, 21(9):2655-2671.

41. Yang $X T$, Zhang ZQ, Joyce D, Huang XM, Xu LY, Pang XQ: Characterization of chlorophyll degradation in banana and plantain during ripening at high temperature. Food Chemistry 2009, 114(2):383-390.

42. Chen K, Zhang F, Kan J: Characterization of chlorophyll breakdown in green prickleyashes (Zanthoxylum schinifolium Zucc.) during slow drying. Eur Food Res Technol 2012, 234(6):1023-1031.

43. Meskauskiene R, Nater M, Goslings D, Kessler F, op den Camp R, Apel K: FLU: a negative regulator of chlorophyll biosynthesis in Arabidopsis thaliana. Proc Natl Acad Sci USA 2001, 98(22):12826-12831.

44. Kleffmann T, Russenberger D, von Zychlinski A, Christopher W, Sjolander K, Gruissem W, Baginsky S: The Arabidopsis thaliana chloroplast proteome reveals pathway abundance and novel protein functions. Curr Biol 2004, 14(5):354-362.

45. Punta M, Coggill PC, Eberhardt RY, Mistry J, Tate J, Boursnell C, Pang N, Forslund K, Ceric G, Clements J, Heger A, Holm L, Sonnhammer EL, Eddy SR, Bateman A, Finn RD: The Pfam protein families database. Nucleic Acids Res 2012, 40(Database issue):D290-301.

46. Langmead B, Trapnell C, Pop M, Salzberg SL: Ultrafast and memoryefficient alignment of short DNA sequences to the human genome. Genome Biol 2009, 10(3):R25. 
47. Chen YC, Hsiao CD, Lin WD, Hu CM, Hwang PP, Ho JM: ZooDDD: a crossspecies database for digital differential display analysis. Bioinformatics 2006, 22(17):2180-2182.

48. Audic S, Claverie JM: The significance of digital gene expression profiles. Genome Res 1997, 7(10):986-995.

49. Romualdi C, Bortoluzzi S, D'Alessi F, Danieli GA: IDEG6: a web tool for detection of differentially expressed genes in multiple tag sampling experiments. Physiol Genomics 2003, 12(2):159-162.

50. Benjamini Y, Drai D, Elmer G, Kafkafi N, Golani I: Controlling the false discovery rate in behavior genetics research. Behav Brain Res 2001, 125(1-2):279-284.

51. Saldanha AJ: Java Treeview-extensible visualization of microarray data. Bioinformatics 2004, 20(17):3246-3248.

52. Cline MS, Smoot M, Cerami E, Kuchinsky A, Landys N, Workman C, Christmas R, Avila-Campilo I, Creech M, Gross B, Hanspers K, Isserlin R, Kelley R, Killcoyne S, Lotia S, Maere S, Morris J, Ono K, Pavlovic V, Pico AR, Vailaya A, Wang PL, Adler A, Conklin BR, Hood L, Kuiper M, Sander C, Schmulevich I, Schwikowski B, Warner $G J$, et al: Integration of biological networks and gene expression data using Cytoscape. Nat Protoc 2007, 2(10):2366-2382.

53. Chen $H$, Boutros PC: VennDiagram: a package for the generation of highly-customizable Venn and Euler diagrams in R. BMC Bioinformatics 2011, 12:35.

54. Xiao D, Zhang NW, Zhao JJ, Bonnema G, Hou XL: Validation of reference genes for real-time quantitative PCR normalisation in non-heading Chinese cabbage. Funct Plant Biol 2012, 39(4):342-350.

55. Pfaffl MW: A new mathematical model for relative quantification in real-time RT-PCR. Nucleic Acids Res 2001, 29(9):e45.

56. Schneider M, Consortium TU, Poux S: UniProtKB amid the turmoil of plant proteomics research. Front Plant Sci 2012, 3:270.

57. O'Donovan C, Martin MJ, Gattiker A, Gasteiger E, Bairoch A, Apweiler R: High-quality protein knowledge resource: SWISS-PROT and TrEMBL. Brief Bioinform 2002, 3(3):275-284.

58. Ashburner M, Ball CA, Blake JA, Botstein D, Butler $H$, Cherry JM, Davis AP, Dolinski K, Dwight SS, Eppig JT, Harris MA, Hill DP, Issel-Tarver L, Kasarskis A, Lewis S, Matese JC, Richardson JE, Ringwald M, Rubin GM, Sherlock G, Consortium GO: Gene ontology: tool for the unification of biology. The Gene Ontology Consortium. Nat Genet 2000, 25(1):25-29.

59. Tanabe M, Kanehisa M: Using the KEGG database resource. Curr Protoc Bioinformatics 2012, 1:1-12.

60. Tatusov RL, Koonin EV, Lipman DJ: A genomic perspective on protein families. Science 1997, 278(5338):631-637.

61. Li L, Stoeckert CJ Jr, Roos DS: OrthoMCL: identification of ortholog groups for eukaryotic genomes. Genome Res 2003, 13(9):2178-2189.

62. Tang H, Bowers JE, Wang X, Ming R, Alam M, Paterson AH: Synteny and collinearity in plant genomes. Science 2008, 320(5875):486-488.

doi:10.1186/1471-2229-14-71

Cite this article as: Song et al.: Genes associated with agronomic traits in non-heading Chinese cabbage identified by expression profiling. BMC Plant Biology 2014 14:71.

\section{Submit your next manuscript to BioMed Central and take full advantage of:}

- Convenient online submission

- Thorough peer review

- No space constraints or color figure charges

- Immediate publication on acceptance

- Inclusion in PubMed, CAS, Scopus and Google Scholar

- Research which is freely available for redistribution 